\title{
Frequency and risk factors of impaired left ventricular global longitudinal strain in patients with end-stage renal disease: a two-dimensional speckle-tracking echocardiographic study
}

\author{
Fengzhen Liu, Xiaolin Wang, Dan Liu, Chunquan Zhang \\ Department of Ultrasound, the Second Affiliated Hospital of Nanchang University, Nanchang, China \\ Correspondence to: Chunquan Zhang. Department of Ultrasound, the Second Affiliated Hospital of Nanchang University, 1 Minde Road, Donghu \\ District, Nanchang 330006, China. Email: doctorCQZhang@163.com.
}

\begin{abstract}
Background: It has been identified that two-dimensional speckle-tracking imaging (2D-STI) enables the early detection of left ventricular (LV) systolic dysfunction. This study's objective was to evaluate the frequency of impaired LV global longitudinal strain (GLS) and investigate the factors in end-stage renal disease (ESRD) patients with preserved LV ejection fraction (LVEF) associated with the impaired GLS.

Methods: A total of 100 ESRD patients with preserved LVEF who underwent transthoracic echocardiography (TTE) were studied. The GLS was calculated as the average of peak longitudinal strain from 18 myocardial segments obtained utilizing the three-standard apical imagings. According to a predefined cutoff, a GLS absolute value of less than $18 \%$ was considered subclinical LV systolic dysfunction. Results: Impaired LV GLS $<18 \%$ was detected in 58 participants (58/100, 58\%). Multivariate analysis exhibited that increased LV mass index was independently associated with impaired GLS $<18 \%$ [odds ratio (OR): 1.028, 95\% confidence interval (CI): 1.004-1.052, $\mathrm{P}=0.020]$. For sequential logistic regression models, model 1 , based on parameters included in multivariate logistic regression $\left(\chi^{2}=30.0\right)$, was improved by the addition of the $L V$ mass index $\left(\chi^{2}=37.4, \mathrm{P}<0.01\right)$.
\end{abstract}

Conclusions: The frequency of impaired LV GLS in ESRD patients with preserved LVEF was relatively high. An increased LVEF was independently associated with impaired LV GLS.

Keywords: End-stage renal disease (ESRD); speckle-tracking imaging (STI); strain

Submitted Sep 04, 2020. Accepted for publication Jan 11, 2021.

doi: 10.21037/qims-20-1034

View this article at: http://dx.doi.org/10.21037/qims-20-1034

\section{Introduction}

Patients with end-stage renal disease (ESRD) are highly prone to cardiovascular diseases (CVDs) in comparison with the general population $(1,2)$, and more than $60 \%$ of ESRD deaths are caused by CVD such as malignant arrhythmia, chronic heart failure, sudden cardiac death, and so on. Left ventricular (LV) dysfunction is one of the leading causes of cardiac death, which is common in patients with ESRD. Therefore, early detection of LV dysfunction in these patients is vitally necessary. However, conventional echocardiography is not sensitive in detecting early deterioration of cardiac function as global LV parameters of the cardiac cavity size and ejection fraction (EF) are generally normal, despite the presence of subtle LV systolic dysfunction (3).

Myocardial strain acquired by two-dimensional speckletracking imaging (2D-STI) has emerged in the last decade as a reliable technique for detecting subclinical LV systolic dysfunction, which has allowed advancements in LV quantification beyond LVEF (4). Moreover, global longitudinal systolic strain (GLS), derived from 2D-STI, has been demonstrated as a superior predictor of adverse cardiovascular outcomes and all-cause mortality 
with advanced kidney disease compared to LVEF (5-7). Nonetheless, the question has arisen regarding what ESRD patients' factors are related to impaired LV systolic longitudinal strain. Accordingly, this study's objective was to evaluate the factors in ESRD patients with preserved LVEF associated with impaired LV longitudinal systolic function.

\section{Methods}

\section{Study population}

A total of 112 inpatients and outpatients of our hospital with ESRD were recruited from September 2019 to May 2020. The inclusion criteria were as follows: $>18$ years old; estimated glomerular filtration rate (eGFR) of $\leq 15 \mathrm{~mL} / \mathrm{min} / 1.73 \mathrm{~m}^{2}$ by the Modification of Diet in Renal Disease formula (8) or on maintenance hemodialysis, LVEF $>50 \%$ normal sinus rhythm. There were 12 patients excluded due to coronary artery disease $(n=7)$, severe valve diseases $(n=2)$, and suboptimal image quality $(n=3)$. Therefore, 100 participants were finally included in this study. The Ethics Committee approved the study of the Second Affiliated Hospital of Nanchang University, and individual consent for this retrospective analysis was waived. This study was performed following the World Medical Association Declaration of Helsinki.

\section{Conventional transthoracic echocardiography (TTE)}

Comprehensive conventional TTE was performed by welltrained cardiologists in the left lateral decubitus position for optimal image quality, using a commercially available GE Healthcare Vivid E95 equipped with M5S $3.5 \mathrm{mHz}$ transducer (GE Healthcare, Chicago, IL, USA). The dimension of LV at end-diastole (LVEDD) and end-systole (LVESD), interventricular septum thickness in diastole (IVSd), and posterior wall thickness (PWTd) were measured by M-mode echocardiography from the parasternal longaxis view. The $L V$ mass was determined using the Devereux formula and was indexed by body surface area (BSA) for LV mass index (LVMI). Relative wall thickness (RWT) was calculated as the ratio of $2 \times$ PWTd/LVEDD. Hypertrophy of the $\mathrm{LV}(\mathrm{LVH})$ was defined as LVMI (male) $>115 \mathrm{~g} / \mathrm{m}^{2}$ and LVMI (female) $>95 \mathrm{~g} / \mathrm{m}^{2}$ (9). The LVEF was determined by utilizing biplane Simpson's method. From the apical 4-chamber view, mitral peak early diastolic velocity (E) and peak late diastolic velocity (A) were assessed by pulsed-wave Doppler. Tissue Doppler assessed the early diastolic peak velocity (e') at the septal mitral annulus site. The ratio (E/e') 73 was calculated as a reliable index of LV filling pressures 74 (10-12). All measurements were completed according 75 to the guidelines published by the American Society of 76 Echocardiography (13).

\section{$2 D S T I$}

To perform the speckle tracking strain analysis, three standard apical views (4-chamber, 2-chamber, and 3 -chamber) were acquired by grayscale images at frame rates of 50-80 frames/sec and were stored in a cineloop format. The analysis was executed online using GE propriety software (GE, Vingmed Ultrasound, Horten, Norway). Detailed information on the methodological procedure of 2D-STI has been previously described (14). In short, the software automatically determined the endocardial and epicardial boundaries at end-systole with automated function imaging (AFI) for each view, with adjustments performed manually until optimal tracking was achieved. The software automatically divided the LV into six segments in each view for 18 segments. Then, GLS was determined as the average of peak longitudinal strain for all the segments within the three standard apical views. For this study, GLS was represented as an absolute value. Participants were excluded if there was any suboptimal segment. As previously reported, a GLS absolute value of $<18 \%$ was considered subclinical LV systolic dysfunction in participants with preserved LVEF, according to previous researches $(9,12,15)$.

\section{Statistical analysis}

Data were expressed as mean \pm standard deviation (SD) and frequencies (\%) for continuous variables and categorical variables, respectively. Comparisons of continuous variables in two groups were performed using the Student's $t$-test or Mann-Whitney $U$ test. Categorical variables were compared by $\chi^{2}$ or Fisher's exact test. Pearson correlation coefficients were used to assess the correlation between two variables. Univariate logistic regression was used to determine possible correlations of reduced LV systolic function (GLS $<18 \%$ ), and parameters with $\mathrm{P}$ value $<0.1$ or clinically relevant ones on univariate analysis were incorporated into the multivariate analysis to determine the independent associations $(\mathrm{P}<0.05)$. Multicollinearity between variables was not be allowed in the multivariate analysis. A sequential logistic model for GLS $<18 \%$ was introduced and tested by 


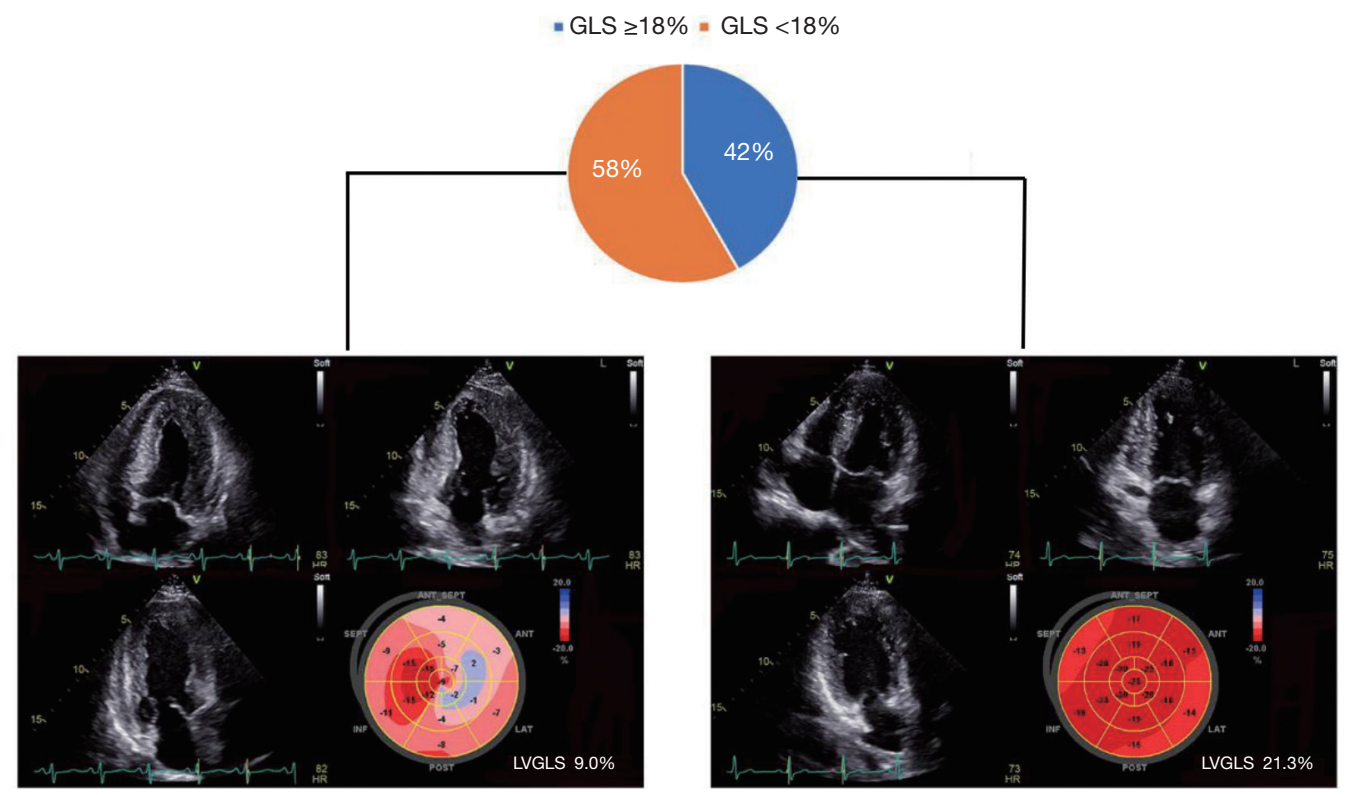

Figure 1 Prevalence of LV systolic dysfunction in terms of LV GLS in ESRD patients with preserved LVEF. Fifty-eight percent of the patients showed LV systolic dysfunction. The patient on the left-side exhibited severely impaired LV GLS, the patient on the right-side showed normal LV GLS. GLS is expressed as absolute values. LV, left ventricular; GLS, global longitudinal strain; ESRD, end-stage renal disease; LVEF, LV ejection fraction.

$\chi^{2}$ tests to determine the incremental value of independent risk factors. All tests were two-sided, and $\mathrm{P}$ value $<0.05$ was considered to indicate statistical significance. All statistical analyses were processed with the software SPSS version 26.0 (IBM, SPSS Inc., Chicago, IL, USA).

\section{Results}

\section{Clinical and laboratory characteristics}

Of 100 ESRD participants (aged $52.3 \pm 15.1$ years, $68 \%$ male) with LVEF $\geq 50 \%, 42(42 / 100,42 \%)$ had LV GLS $\geq 18 \%$ and $58(58 / 100,58 \%)$ showed LV GLS $<18 \%$ (Figure 1). The baseline clinical characteristics of the two groups are depicted in Table 1. There were no inter-group differences for age, gender, body mass index, or BSA $(\mathrm{P}>0.05)$. Participants with LV GLS $<18 \%$ had higher systolic blood pressure (SBP), diastolic blood pressure (DBP), and lower eGFR compared to those with LV GLS $\geq 18 \%$. Compared to participants with $\mathrm{LV}$ GLS $\geq 18 \%$, heart rate (HR), serum creatinine, phosphorus, and product of calcium-phosphorus $(\mathrm{Ca} \times \mathrm{P})$ in those with GLS $<18 \%$ tended to be higher, but the differences were not statistically significant.

\section{Echocardiographic parameters for patients with GLS} $<18 \%$ and $G L S \geq 18 \%$

Compared to LV GLS $\geq 18 \%$ participants, conventional parameters in IVSd, PWTd, LVEDD, LVESD, LVEF, RWT, LVMI, and E/e' were significantly aggravated in patients with a GLS $<18 \%$. Additionally, the prevalence of $\mathrm{LVH}$ in participants with GLS $<18 \%$ was relatively high. Among the 68 ESRD participants with LVH, 50\% (21/42) of those with GLS $\geq 18 \%$ had LVH, and $81 \%(47 / 58)$ of those with GLS $<18 \%$ showed LVH. Compared to ESRD participants with non-LVH, GLS was significantly decreased in those with $\mathrm{LVH}(19.0 \% \pm 2.4 \%$ vs. $16.4 \% \pm 3.2 \%, \mathrm{P}<0.001)$ (Table 2).

\section{Association of LV geometry and clinical parameters with GLS}

Linear correlation analyses showed that GLS correlated significantly with LVMI $(\mathrm{r}=-0.576, \mathrm{P}<0.001)$, eGFR $(r=0.284, \mathrm{P}<0.01)$, LVEF $(r=0.468, \mathrm{P}<0.01)$, LVEDD $(r=-0.328, \mathrm{P}<0.01)$, LVESD $(\mathrm{r}=-0.467, \mathrm{P}<0.01)$, and RWT $(r=-0.373, P<0.01)$ in all participants. With the increase of 
Table 1 Baseline characteristics and laboratory variables

\begin{tabular}{|c|c|c|c|c|}
\hline Variables & ESRD & GLS $\geq 18 \%(n=42)$ & GLS <18\% (n=58) & $P$ value \\
\hline Age, year & $52 \pm 15$ & $55 \pm 14$ & $50 \pm 16$ & 0.160 \\
\hline Gender & 32 [32] & 12 [29] & 20 [35] & 0.532 \\
\hline Body mass index, $\mathrm{kg} / \mathrm{m}^{2}$ & $21.98 \pm 3.46$ & $21.87 \pm 3.22$ & $22.06 \pm 3.65$ & 0.778 \\
\hline $\mathrm{HR}$, beats/min & $78 \pm 13$ & $75 \pm 12$ & $80 \pm 14$ & 0.054 \\
\hline SBP, mmHg & $147 \pm 26$ & $136 \pm 24$ & $155 \pm 25$ & $<0.001$ \\
\hline DBP, $\mathrm{mmHg}$ & $87 \pm 16$ & $82 \pm 15$ & $90 \pm 17$ & 0.022 \\
\hline Hemodialysis & $35[35]$ & $15[36]$ & 20 [35] & 0.899 \\
\hline \multicolumn{5}{|l|}{ Medications } \\
\hline ACE inhibitor/ARB & $10[10]$ & $3[7]$ & 7 [12] & 0.418 \\
\hline$\beta$-blocker & $20[20]$ & $9[21]$ & $11[19]$ & 0.761 \\
\hline$\alpha$-blocker & $8[8]$ & $5[12]$ & $3[5]$ & 0.221 \\
\hline Calcium antagonist & $53[53]$ & $22[52]$ & $31[53]$ & 0.916 \\
\hline Diuretic & $27[27]$ & 12 [29] & $10[17]$ & 0.177 \\
\hline \multicolumn{5}{|l|}{ Laboratory results } \\
\hline Serum creatinine, $\mu \mathrm{mol} / \mathrm{L}$ & $869.06 \pm 361.21$ & $800.13 \pm 340.07$ & $918.98 \pm 370.66$ & 0.105 \\
\hline Calcium-phosphor, $\mathrm{mmol}^{2} / \mathrm{L}^{2}$ & $3.80 \pm 1.24$ & $3.55 \pm 1.00$ & $3.98 \pm 1.37$ & 0.093 \\
\hline
\end{tabular}

Continuous data are presented as mean \pm SD. Categorical data are presented as numbers [\%]. GLS is expressed as absolute values. ESRD, end-stage renal disease; GLS, global longitudinal strain; BSA, body surface area; HR, heart rate; SBP, systolic blood pressure; DBP, diastolic blood pressure; ACE, angiotensin-converting enzyme; ARB, angiotensin receptor blocker; eGFR, estimated glomerular filtration rate; $\mathrm{SD}$, standard deviation.

LVMI, the absolute values of GLS decreased accordingly (Figure 2).

\section{Related factors of reduced GLS for ESRD patients}

Univariable and multivariable analyses were performed to determine independent factors associated with impaired GLS (Table 3). In univariable analysis, SBP, DBP, eGFR,
LVEF, LVEDD, LVESD, IVSd, PWTd, LVMI, and E/e' 175 ratio were associated with GLS $<18 \%$. Parameters with 176 $\mathrm{P}$ value $<0.1$ or clinical relevance on univariate analysis 177 were incorporated into the multivariate analysis. To 178 avoid multicollinearity, IVSd, PWTd, and LVEDD were 179 not included in the multivariable analysis. By further 180 multivariate analysis, an increased LVMI was independently 181 associated with GLS $<18 \%$ [odds ratio (OR): 1.028; 182 
Table 2 Echocardiographic variables in patients with ESRD

\begin{tabular}{|c|c|c|c|c|}
\hline Variables & ESRD & GLS $\geq 18 \%(n=42)$ & GLS $<18 \%(n=58)$ & $P$ value \\
\hline PWTd, mm & $10.94 \pm 1.89$ & $10.05 \pm 1.29$ & $11.59 \pm 2.00$ & $<0.001$ \\
\hline LVEDD, $\mathrm{mm}$ & $49.19 \pm 5.01$ & $47.86 \pm 4.91$ & $50.16 \pm 4.91$ & 0.023 \\
\hline LVESD, mm & $32.21 \pm 4.86$ & $30.43 \pm 4.38$ & $33.50 \pm 4.82$ & 0.002 \\
\hline LVMI, $g / \mathrm{m}^{2}$ & $129.57 \pm 42.59$ & $110.00 \pm 24.26$ & $143.75 \pm 47.33$ & $<0.001$ \\
\hline LVH, n [\%] & 68 [68] & $21[50]$ & $47[81]$ & 0.001 \\
\hline LVEF, \% & $62.29 \pm 6.17$ & $64.60 \pm 6.15$ & $60.62 \pm 5.68$ & 0.001 \\
\hline$E / e^{\prime}$ & $13.21 \pm 3.75$ & $11.77 \pm 3.26$ & $14.26 \pm 3.76$ & 0.001 \\
\hline
\end{tabular}

Continuous data are presented as mean \pm SD. Categorical data are presented as numbers [\%]. GLS is expressed as absolute values. ESRD, end-stage renal disease; GLS, global longitudinal strain; IVSd, interventricular septum thickness in diastole; PWTd, posterior wall thickness in diastole; LVEDD, left ventricular end-diastolic diameter; LVESD, left ventricular end-systolic diameter; RWT, relative wall thickness; LVMI, left ventricular mass index; LVH, left ventricular hypertrophy; LVEF, left ventricular ejection fraction; E/e', peak early diastolic velocity (by pulsed-wave Doppler)/early diastolic peak velocity (by tissue Doppler); SD, standard deviation.

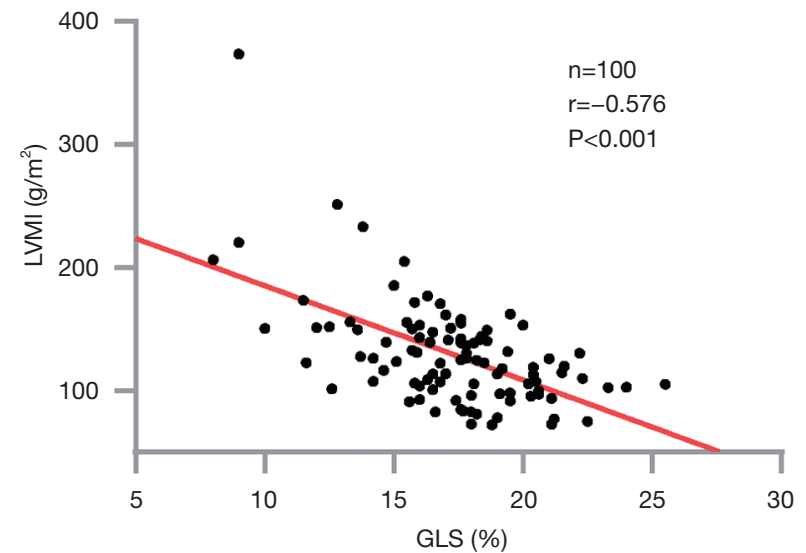

Figure 2 Linear correlation analyses. LV GLS decreased significantly with LVMI in patients. GLS is expressed as absolute values. LV GLS, left ventricular global longitudinal strain; LVMI, LV mass index.
95\% confidence interval (CI): 1.004-1.052, $\mathrm{P}=0.020]$. A sequential logistic analysis for predicting GLS $<18 \%$ was done, starting with multivariate logistic regression (model 1: SBP, DBP, eGFR, LVEF, LVESD, E/e'), and LVMI was added to model 1 via stepwise block analysis to assess its incremental value. Model $1\left(\chi^{2}=30.0\right)$ was improved by the addition of LVMI $\left(\chi^{2}=37.4, \mathrm{P}<0.01\right)$, as shown in Figure 3 .
Discussion 190

Main findings

The present study's major finding was that the frequency of LV systolic dysfunction in ESRD participants with preserved LVEF was relatively high. Over half (58\%) of ESRD participants showed LV systolic dysfunction, defined by LV GLS $<18 \%$ in normal LVEF. Compared to participants with LV GLS $\geq 18 \%, \mathrm{LV}$ geometry was larger or thicker in those with LV GLS $<18 \%$. An increased LVMI was independently associated with abnormal GLS, which was consistent with previous studies $(6,16,17)$.

\section{Impaired LV GLS in ESRD patients without overt heart} failure

Our study exhibited a high proportion of impaired GLS, 207 with 58\% (58/100) incidence among ESRD patients, which 208 was similar to previous reports describing impaired LV 209 GLS in ESRD patients with preserved LVEF. In a study 210 by Huang et al. study (7), 42\% (80/190) of hemodialysis 211 participants had LV longitudinal systolic dysfunction defined 212 as GLS $<16 \%$. Similarly, Rhea et al. reported 47\% (66/139) 213 of advanced chronic kidney disease (CKD) participants 214 as impaired GLS (determined as GLS <18\%) (15). 215 
Table 3 Univariate and multivariate logistic regression analysis for detecting GLS $<18 \%$ ESRD patients with preserved LVEF

\begin{tabular}{|c|c|c|c|c|}
\hline Variables & \multicolumn{2}{|c|}{ Univariate } & \multicolumn{2}{|c|}{ Multivariate } \\
\hline eGFR, $\mathrm{mL} / \mathrm{min} / 1.73 \mathrm{~m}^{2}$ & $0.877(0.785-0.987)$ & 0.022 & $0.985(0.864-1.123)$ & 0.819 \\
\hline $\mathrm{SBP}, \mathrm{mmHg}$ & $1.032(1.013-1.051)$ & 0.001 & $1.005(0.971-1.041)$ & 0.775 \\
\hline $\mathrm{DBP}, \mathrm{mmHg}$ & $1.031(1.004-1.059)$ & 0.026 & $1.035(0.980-1.093)$ & 0.213 \\
\hline IVSd, mm & 1.739 (1.289-2.346) & $<0.001$ & - & - \\
\hline PWTd, mm & $1.924(1.392-2.660)$ & $<0.001$ & - & - \\
\hline LVEDD, mm & $1.103(1.011-1.204)$ & 0.027 & - & - \\
\hline LVESD, mm & $1.158(1.052-1.276)$ & 0.003 & $0.909(0.744-1.110)$ & 0.350 \\
\hline$E / e^{\prime}$ & $1.239(1.083-1.417)$ & 0.002 & $1.160(0.972-1.384)$ & 0.099 \\
\hline
\end{tabular}

GLS is expressed as absolute values. GLS, global longitudinal strain; ESRD, end-stage renal disease; LVEF, left ventricular ejection fraction; OR, odds ratio; Cl, confidence interval; SBP, systolic blood pressure; DBP, diastolic blood pressure; HR, heart rate; IVSd, interventricular septum thickness in diastole; PWTd, posterior wall thickness in diastole; LVEDD, left ventricular end-diastolic diameter; LVESD, left ventricular end-systolic diameter; LVMI, left ventricular mass index; E/e', peak early diastolic velocity (by pulsed-wave Doppler)/early diastolic peak velocity (by tissue Doppler).

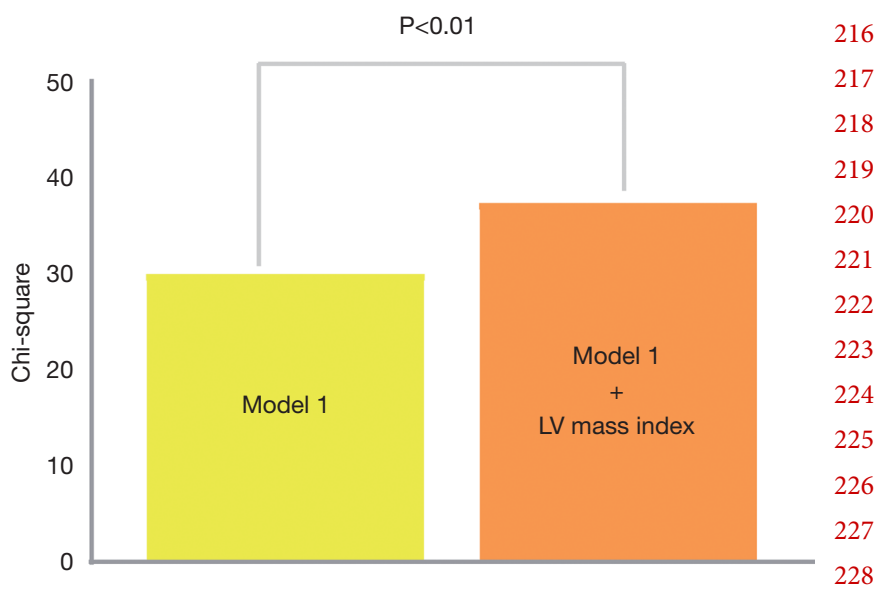

Figure 3 Incremental value by sequential logistic regression 229 models for identifying LV GLS $<18 \%$, presented as global $\chi^{2} 230$ values. Model 1 (SBP, DBP, eGFR, LVEF, LVESD, E/e') based ${ }_{231}$ on significant univariate logistic regression parameters $\left(\chi^{2}=30.0\right) 232$ was improved by the addition of LVMI $\left(\chi^{2}=37.4, \mathrm{P}<0.01\right)$. GLS 233 is expressed as absolute values. LV GLS, left ventricular global 234 longitudinal strain; SBP, systolic blood pressure; DBP, diastolic 235 blood pressure; eGFR, estimated glomerular filtration rate; E/e', 236 peak early diastolic velocity (by pulsed-wave Doppler)/early 237 diastolic peak velocity (by tissue Doppler); LVEF, LV ejection 238 fraction; LVESD, LV end-systolic diameter; LVMI, LV mass index. 239
The difference in the incidence of abnormal GLS in CKD patients with retained LVEF may depend on patient characteristics, such as the severity of renal function and dialysis availability. The main pathophysiological explanation may be that LV longitudinal dysfunction reflected early CKD-related myocardial changes such as myocardial ischemia, hypertrophy, and fibrosis because subendocardial longitudinal myocardial fibers were susceptible to reduced coronary perfusion and increased wall stress $(18,19)$. Similarly, GLS was also shown to enable the detection of subclinical myocardial dysfunction of many CVDs, including hypertension and ischemic heart diseases (20).

\section{Related risk factors of LV longitudinal systolic myocardial dysfunction}

In our study, increased LVMI was independently associated with abnormal GLS in ESRD participants with preserved LVEF, which was concordant with previous studies $(6,16,17)$. The increase of LVMI suggested varying degrees of LVH, which was a common sequela in ESRD. Wang et al. and Nardi et al. have reported the prevalence of $\mathrm{LVH}$ as $74.5 \%$ and $73.7 \%$ of ESRD patients, respectively $(17,21)$. 
The development of LVH was virtually attributed to the presence of all of these factors (arterial hypertension, volume expansion, calcium-phosphate metabolic abnormalities, arterial stiffness, anemia, and activation of the renin-angiotensin-aldosterone system) (22). As expected, in our analysis of $68(68 \%)$ cases of ESRD with $\mathrm{LVH}$, the prevalence of LVH in patients with GLS $<18 \%$ was relatively higher than that of GLS $\geq 18 \%(50 \%$ vs. $81 \%)$. Compared to ESRD patients with non-LVH, GLS was significantly decreased in patients with LVH, suggesting that reduced GLS was closely associated with LVH. From the pathophysiologic standpoint, the close correlation between decreased GLS in ESRD and larger LVH/LVMI can be explained as follows: pathological myocardial hypertrophy induced the activation of cellular apoptotic and autophagic signals that increased collagen production within the extracellular matrix (ECM), leading to myocardial fibrosis (MF) (23). Increasing levels of MF led to ventricular stiffening, thereby impairing the systolic and diastolic function. In contrast, higher blood pressure (higher afterload) was not an independent factor for detecting GLS $<18 \%$, although it frequently existed in ESRD. The reason was most likely that hypertension was not the only driver in reducing GLS in patients with ESRD. In early-stage CKD, higher blood pressure remained the driving force behind the development of LV systolic dysfunction, but its influence diminished as the CKD stage progressed to ESRD (24).

\section{Clinical implications}

Our findings indicated that a significant proportion of ESRD patients without overt heart failure have asymptomatic systolic longitudinal myocardial dysfunction. This high proportion of cardiac abnormalities requires close follow-up to detect the early development of heart failure and strictly control cardiovascular risk factors, including rigorous control of blood pressure for more stable and tightly controlled volume load, and early application of drugs to reduce MF [angiotensin-converting enzyme (ACE) inhibitors and $\beta$-blockers] to improve ventricular remodeling. Guidelines recommend an echocardiogram for all dialysis patients 1-3 months after renal replacement therapy initiation and every 3 years subsequently (25). Our opinion is that all newly admitted ESRD patients should undergo a comprehensive echocardiographic examination including GLS, and follow-up with serial studies at shorter intervals of 12-18 months should be necessitated as it seems to add prognostic value $(26,27)$. Also, we believe that repeat echocardiography after about 6 months is useful for risk 288 stratification with symptomatic changes, new clinical events, or treatment likely to affect cardiac function (27). It remains to be studied whether shorter repeated echocardiographic monitoring, including GLS, conveys comparable prognostic information.

\section{Limitations}

This study had some limitations. First, there were a relatively small number of ESRD participants from a single study center. Second, the study was cross-sectional, making the findings' prognostic significance unclear and requiring further verification. Finally, the study did not include a healthy control group. A more comprehensive analysis may be needed.

\section{Conclusions}

Our study confirmed that reduced GLS frequently occurs in patients with ESRD. Moreover, increased LVMI was shown to be significantly associated with abnormal LV longitudinal systolic function.

\section{Acknowledgments}

Funding: This study was supported by grants from the Key Science and Technology Research Project of the Department of Education, Jiangxi Province (190002), and the Postgraduate Innovation Special Fund project of Nanchang University (CX 2019135).

\section{Footnote}

Conflicts of Interest: All authors have completed the ICMJE uniform disclosure form (available at http://dx.doi. org/10.21037/qims-20-1034). The authors have no conflicts of interest to declare.

Ethical Statement: This study was approved by the Ethics Committee of the Second Affiliated Hospital of Nanchang University and individual consent for this retrospective analysis was waived. This study was performed in accordance with the World Medical Association Declaration of Helsinki.

Open Access Statement: This is an Open Access article distributed in accordance with the Creative Commons 
Attribution-NonCommercial-NoDerivs 4.0 International License (CC BY-NC-ND 4.0), which permits the noncommercial replication and distribution of the article with the strict proviso that no changes or edits are made and the original work is properly cited (including links to both the formal publication through the relevant DOI and the license). See: https://creativecommons.org/licenses/by-nc-nd/4.0/.

\section{References}

1. Essig M, Escoubet B, de Zuttere D, Blanchet F, Arnoult F, Dupuis E, Michel C, Mignon F, Mentre F, Clerici C, Vrtovsnik F. Cardiovascular remodelling and extracellular fluid excess in early stages of chronic kidney disease. Nephrol Dial Transplant 2008;23:239-48.

2. Sabatino A, D'Alessandro C, Regolisti G, di Mario F, Guglielmi G, Bazzocchi A, Fiaccadori E. Muscle mass assessment in renal disease: the role of imaging techniques. Quant Imaging Med Surg 2020;10:1672-86.

3. Unger ED, Dubin RF, Deo R, Daruwalla V, Friedman JL, Medina C, Beussink L, Freed BH, Shah SJ. Association of chronic kidney disease with abnormal cardiac mechanics and adverse outcomes in patients with heart failure and preserved ejection fraction. Eur J Heart Fail 2016;18:103-12.

4. Chan J, Shiino K, Obonyo NG, Hanna J, Chamberlain R, Small A, Scalia IG, Scalia W, Yamada A, HamiltonCraig CR, Scalia GM, Zamorano JL. Left ventricular global strain analysis by two-dimensional speckletracking echocardiography: the learning curve. J Am Soc Echocardiogr 2017;30:1081-90.

5. Krishnasamy R, Isbel NM, Hawley CM, Pascoe EM, Burrage M, Leano R, Haluska BA, Marwick TH, Stanton T. Left ventricular global longitudinal strain (GLS) is a superior predictor of all-cause and cardiovascular mortality when compared to ejection fraction in advanced chronic kidney disease. PLoS One 2015;10:e0127044.

6. Hensen LCR, Goossens K, Delgado V, Abou R, Rotmans JI, Jukema JW, Bax JJ. Prevalence of left ventricular systolic dysfunction in pre-dialysis and dialysis patients with preserved left ventricular ejection fraction. Eur J Heart Fail 2018;20:560-8.

7. Huang JC, Su HM, Wu PY, Lee JJ, Lee WH, Chen SC, Chiu YW, Hsu YL, Chang JM, Chen HC. Ratio of early mitral inflow velocity to the global diastolic strain rate and global left ventricular longitudinal systolic strain predict overall mortality and major adverse cardiovascular events in hemodialysis patients. Dis Markers 2019;2019:7512805.
8. Levey AS, Bosch JP, Lewis JB, Greene T, Rogers N, Roth D. A more accurate method to estimate glomerular filtration rate from serum creatinine: a new prediction equation. Modification of Diet in Renal Disease Study Group. Ann Intern Med 1999;130:461-70.

9. Lang RM, Badano LP, Mor-Avi V, Afilalo J, Armstrong A, Ernande L, Flachskampf FA, Foster E, Goldstein SA, Kuznetsova T, Lancellotti P, Muraru D, Picard MH, Rietzschel ER, Rudski L, Spencer KT, Tsang W, Voigt JU. Recommendations for cardiac chamber quantification by echocardiography in adults: an update from the American Society of Echocardiography and the European Association of Cardiovascular Imaging. Eur Heart J Cardiovasc Imaging 2015;16:233-70.

10. Li J, Li A, Wang J, Zhang Y, Zhou Y. Early left ventricular dysfunction detected by speckle tracking in longterm hemodialysis patients with valvular calcification. Cardiorenal Med 2019;9:22-30.

11. Huang WM, Lin YP, Chen CH, Yu WC. Tissue Doppler imaging predicts outcomes in hemodialysis patients with preserved left ventricular function. J Chin Med Assoc 2019;82:351-5.

12. Mochizuki Y, Tanaka H, Matsumoto K, Sano H, Toki H, Shimoura H, Ooka J, Sawa T, Motoji Y, Ryo K, Hirota Y, Ogawa W, Hirata K. Clinical features of subclinical left ventricular systolic dysfunction in patients with diabetes mellitus. Cardiovasc Diabetol 2015;14:37.

13. Nagueh SF, Smiseth OA, Appleton CP, Byrd BF 3rd, Dokainish H, Edvardsen T, Flachskampf FA, Gillebert TC, Klein AL, Lancellotti P, Marino P, Oh JK, Popescu $\mathrm{BA}$, Waggoner AD. Recommendations for the evaluation of left ventricular diastolic function by echocardiography: an update from the American Society of Echocardiography and the European Association of Cardiovascular Imaging. J Am Soc Echocardiogr 2016;29:277-314.

14. Oberhoffer FS, Abdul-Khaliq H, Jung AM, Zemlin M, Rohrer TR, Abd El Rahman M. Assessment of left ventricular myocardial work in Turner syndrome patients: insights from the novel non-invasive pressure-strain loop analysis method. Quant Imaging Med Surg 2020;10:15-25.

15. Rhea IB, Morris K, Sawada S, Feigenbaum H. Prevalence, etiology, and clinical implications of reduced longitudinal systolic strain in renal transplant candidates. Echocardiography 2016;33:1676-82.

16. Ravera M, Rosa GM, Fontanive P, Bussalino E, Dorighi U, Picciotto D, Di Lullo L, Dini FL, Paoletti E. Impaired left ventricular global longitudinal strain among patients with chronic kidney disease and end-stage renal disease and 
renal transplant recipients. Cardiorenal Med 2019;9:61-8.

17. Wang H, Liu J, Yao XD, Li J, Yang Y, Cao TS, Yang B. Multidirectional myocardial systolic function in hemodialysis patients with preserved left ventricular ejection fraction and different left ventricular geometry. Nephrol Dial Transplant 2012;27:4422-9.

18. Cameli M, Mandoli GE, Sciaccaluga C, Mondillo S. More than 10 years of speckle tracking echocardiography: Still a novel technique or a definite tool for clinical practice? Echocardiography 2019;36:958-70.

19. Panoulas VF, Sulemane S, Konstantinou K, Bratsas A, Elliott SJ, Dawson D, Frankel AH, Nihoyannopoulos P. Early detection of subclinical left ventricular myocardial dysfunction in patients with chronic kidney disease. Eur Heart J Cardiovasc Imaging 2015;16:539-48.

20. Collier P, Phelan D, Klein A. A test in context: myocardial strain measured by speckle-tracking echocardiography. J Am Coll Cardiol 2017;69:1043-56.

21. Nardi E, Palermo A, Mule G, Cusimano P, Cottone S, Cerasola G. Left ventricular hypertrophy and geometry in hypertensive patients with chronic kidney disease. J Hypertens 2009;27:633-41.

22. Paoletti E, De Nicola L, Gabbai FB, Chiodini P, Ravera M, Pieracci L, Marre S, Cassottana $\mathrm{P}$, Lucà S, Vettoretti S, Borrelli S, Conte G, Minutolo R. Associations of

Cite this article as: Liu F, Wang X, Liu D, Zhang C. Frequency and risk factors of impaired left ventricular global longitudinal strain in patients with end-stage renal disease: a two-dimensional speckle-tracking echocardiographic study. Quant Imaging Med Surg 2021;11(6):2397-2405. doi: 10.21037/qims-20-1034 left ventricular hypertrophy and geometry with adverse $\quad 457$ outcomes in patients with CKD and hypertension. Clin J 458 Am Soc Nephrol 2016;11:271-9.

23. Graham-Brown MP, Patel AS, Stensel DJ, March DS, Marsh AM, McAdam J, McCann GP, Burton JO. Imaging of myocardial fibrosis in patients with end-stage renal disease: current limitations and future possibilities. Biomed Res Int 2017;2017:5453606.

24. Locatelli F, Pozzoni P, Tentori F, del Vecchio L. Epidemiology of cardiovascular risk in patients with chronic kidney disease. Nephrol Dial Transplant 2003;18 Suppl 7:vii2-9.

25. K/DOQI Workgroup. K/DOQI clinical practice guidelines for cardiovascular disease in dialysis patients. Am J Kidney Dis 2005;45:S1-153.

26. Foley RN, Parfrey PS, Kent GM, Harnett JD, Murray 472 DC, Barre PE. Serial change in echocardiographic 473 parameters and cardiac failure in end-stage renal disease. J 474 Am Soc Nephrol 2000;11:912-6.

27. Zoccali C, Benedetto FA, Mallamaci F, Tripepi G, Giacone G, Stancanelli B, Cataliotti A, Malatino LS. Left ventricular mass monitoring in the follow-up of dialysis patients: prognostic value of left ventricular hypertrophy progression. Kidney Int 2004;65:1492-8. 